\title{
The Usefulness of Intraoperative Colonic Irrigation and Primary Anastomosis in Patients Requiring a Left Colon Resection
}

\author{
Youngki Hong, Soomin Nam, Jung Gu Kang \\ Department of Surgery, National Health Insurance Service Ilsan Hospital, Goyang, Korea
}

Purpose: The aim of this study is to assess the short-term outcome of intraoperative colonic irrigation and primary anastomosis and to suggest the usefulness of the procedure when a preoperative mechanical bowel preparation is inappropriate.

Methods: This retrospective study included 38 consecutive patients (19 male patients) who underwent intraoperative colonic irrigation and primary anastomosis for left colon disease between January 2010 and December 2016. The medical records of the patients were reviewed to evaluate the patients' characteristics, operative data, and postoperative short-term outcomes.

Results: Twenty-nine patients had colorectal cancer, 7 patients had perforated diverticulitis, and the remaining 2 patients included 1 with sigmoid volvulus and 1 with a perforated colon due to focal colonic ischemia. A diverting loop ileostomy was created in 4 patients who underwent a low anterior resection. Complications occurred in 15 patients (39.5\%), and the majority was superficial surgical site infections (18.4\%). Anastomotic leakage occurred in one patient (2.6\%) who underwent an anterior resection due sigmoid colon cancer with obstruction. No significant difference in overall postoperative complications and superficial surgical site infections between patients with obstruction and those with peritonitis were noted. No mortality occurred during the first 30 postoperative days. The median hospital stay after surgery was 15 days (range, 8-39 days).

Conclusion: Intraoperative colonic irrigation and primary anastomosis seem safe and feasible in selected patients. This procedure may reduce the burden of colostomy in patients requiring a left colon resection with an inappropriate preoperative mechanical bowel preparation.

Keywords: Intraoperative colonic irrigation; Primary anastomosis; Anastomotic leakage

\section{INTRODUCTION}

The purpose of mechanical bowel preparation is to reduce the rate of postoperative infectious complications by reducing the fe-

Received: April 6, 2017 • Accepted: May 13, 2017

Correspondence to: Jung Gu Kang, M.D.

Department of Surgery, National Health Insurance Service Ilsan Hospital,

100 Ilsan-ro, Ilsandong-gu, Goyang 10444, Korea

Tel: +82-31-900-0216, Fax: +82-31-900-0343

E-mail:kangski@nhimc.or.kr

(c) 2017 The Korean Society of Coloproctology

This is an open-access article distributed under the terms of the Creative Commons Attribution NonCommercial License (http://creativecommons.org/licenses/by-nc/4.0) which permits unrestricted non-

commercial use, distribution, and reproduction in any medium, provided the original work is properly cited. cal load and the bacterial count in the colon. Although some authors reported the adverse effects of mechanical bowel preparation $[1,2]$, it has become a general practice in clinical settings $[3$, 4]. In patients with colon obstruction or peritonitis, however, undergoing preoperative mechanical bowel preparation is not possible. In this situation, general agreement exists that a right hemicolectomy with primary anastomosis can be safely performed for right colon resection $[5,6]$. On the other hand, when it comes to the left colon, several options are available. The Hartmann procedure can be performed as the means of the surgical resection because this procedure does not risk anastomotic leakage. However, the Hartmann procedure has disadvantages for the patients because a second operation is required to reestablish intestinal continuity and many patients may never undergo reversal of a colos- 
tomy $[7,8]$. For these reasons, the Hartmann procedure lays a considerable physical and mental burden on the patients. Self-expanding metallic stents have an established role in the management of left-sided colonic obstruction as a bridge to surgery [911]. However, this procedure can lead to serious bowel perforation in nearly $5 \%$ of the cases [9], and it cannot be performed in cases with peritonitis caused by colon perforation. A 1-stage colectomy with intraoperative colonic irrigation may be another option for treatment in cases where mechanical bowel preparation is not appropriate preoperatively. It is widely accepted as a safe procedure for treatment of left colon obstruction [6]. The purpose of irrigation is to achieve a colon free of feces that allows a decrease in the rate of suture failure and its attendant complications [12]. Thus, the aim of this study is to assess the short-term outcome in patients who underwent a 1 stage colectomy with intraoperative colonic irrigation for left colon resection when preoperative mechanical bowel preparation was inappropriate and to suggest the usefulness of intraoperative colonic irrigation in clinical settings.

\section{METHODS}

\section{Patients}

This retrospective study included 38 consecutive patients who underwent intraoperative colonic irrigation and primary anastomosis for left colon disease between January 2010 and December 2016 in National Health Insurance Corporation Ilsan Hospital, Goyang, Korea. The operations were conducted by 2 surgeons (JGK and YH). The medical records of the patients were collected to evaluate the patients' characteristics, operative data and postoperative short-term outcomes.

Nineteen men and 19 women were treated with intraoperative colonic irrigation during that period. The median age was 74.5 years (range, 46-93 years). Patients with obstruction had a median age of 71 years (range, 47-92 years) whereas those with peritonitis were aged 79 years (range, $46-93$ years). Sixteen patients presented with American Society of Anesthesiologists physical status (ASA PS) classification of 3 or 4 at the time of surgery. Twenty-nine patients had colorectal cancer, and that cancer was located in the descending colon in 2, the sigmoid colon in 22, and the rectum in 5. Seven patients had perforated diverticulitis. The remaining 2 patients included 1 with sigmoid volvulus and 1 with a perforation of the colon due to focal colonic ischemia. The reasons for inadequate mechanical bowel preparation were obstruction in 25 patients (65.8\%) and peritonitis in 13 (34.2\%). Of the 25 patients with obstruction, 21 patients had no definite symptoms of obstruction at admission, and they passed flatus. However, they presented with abdominal distension and pain during mechanical bowel presentation (partial obstruction). In four patients with complete obstruction, insertion of a colonic stent failed or was not feasible prior to surgery. Peritonitis was present in 13 patients. Of these patients, localized peritonitis was identified in 5 patients and generalized peritonitis in 8 by using preoperative computed tomography (Table 1). Twenty-eight patients (73.7\%) presented with one or more associated comorbidity at the time of surgery (Table 2).

The IBM SPSS Statistics ver. 23.0 (IBM Co., Armonk, NY, USA) was used for statistical analyses. Categorical variables were analyzed with the chi-square test, and continuous variables were analyzed with the Mann-Whitney test. A P-value of less than 0.05 was considered statistically significant.

\section{Surgical procedure}

All patients were managed by resection, irrigation, and anastomosis. All laparotomies were performed through a midline incision. The technique used for intraoperative colonic irrigation was similar to that previously described [13]. For intraoperative decompression and irrigation of the colon, a new intraoperative colonic irrigator (NICI, MITech, Seoul, Korea and Dalim BioTech, Seoul, Korea) was used. After mobilization of the left colon, including splenic flexure, in the standard fashion, the segment of the colon was resected. The device was connected to the proximal end of the colon and tightened with the fastening nut to fix it on the end

Table 1. Patient characteristics $(\mathrm{n}=38)$

\begin{tabular}{lc}
\hline Characteristic & Value \\
\hline Sex & $19(50.0)$ \\
Male & $19(50.0)$ \\
Female & $74.5(46-93)$ \\
Age (yr) & \\
ASA PS classification & $2(5.2)$ \\
I & $20(52.6)$ \\
॥ & $14(36.8)$ \\
III & $2(5.2)$ \\
IV & \\
Diagnosis & $29(76.3)$ \\
Cancer & $24(63.2)$ \\
Colon & $5(13.1)$ \\
Rectum & $7(18.4)$ \\
Diverticulitis & $2(5.2)$ \\
Others & \\
Reason for inadequate bowel preparation & $25(65.8)$ \\
Obstruction & $21(55.3)$ \\
Partial obstruction & $4(10.5)$ \\
Complete obstruction & $13(34.2)$ \\
Peritonitis due to bowel perforation & $5(13.2)$ \\
Localized & $8(21.1)$ \\
\hline Generalized & \\
\hline & \\
\hline &
\end{tabular}

Values are presented as number (\%) or median (range).

ASA PS, American Society of Anesthesiologists physical status. 
Table 2. Comorbidity in 38 patients

\begin{tabular}{lc}
\hline Comorbidity & No. (\%) \\
\hline Hypertension & $21(55.7)$ \\
Diabetes & $6(15.8)$ \\
Cardiac disease & $7(18.4)$ \\
Cerebrovascular disease & $5(13.2)$ \\
Chronic obstructive airway disease & $2(5.3)$ \\
End-stage renal disease & $1(2.6)$ \\
Pneumonia & $1(2.6)$ \\
None & $10(26.3)$ \\
\hline
\end{tabular}

of the colon. An irrigation catheter was inserted through a branched tube of the device to the cecum or the hepatic flexure colon. Irrigation was performed with warm saline through the irrigation catheter. After the irrigation had been completed, the irrigation catheter was extracted, and primary anastomosis was done. In patients whose rectum was impacted by stool, rectal irrigation was performed to evacuate the stool before anastomosis.

\section{RESULTS}

The primary anastomosis was performed above the peritoneal reflection of the rectum in 33 patients $(86.8 \%)$ and below the peritoneal reflection of the rectum in $5(13.2 \%)$. Of the latter 5 patients who underwent a low anterior resection, a diverting loop ileostomy was created in four patients. The median operation time of all surgeries was 193.5 minutes (Table 3 ).

Complications occurred in 15 of the 38 patients (39.5\%), and the majority was superficial surgical site infection (18.4\%) (Table 4). Reinterventions were required in 2 patients. Anastomosis leakage after primary anastomosis occurred in 1 patient. He was a 75-year-old man with an ASA PS classification of IV, and he had undergone an anterior resection due to sigmoid colon cancer with obstruction. In this case, the Hartmann operation was performed at postoperative day 4 . The other patient presented with advanced sigmoid colon cancer with invasion to the ureter. An anterior resection combined with a segmental resection of the ureter and an end-to-end anastomosis was done. A ureter stent was placed through the affected ureter at the first operation. Because urine leakage from the ureter anastomotic site was found at postoperative day 2, the ureter stent was repositioned after confirming the malposition of the ureter stent via cystoscopy. Five patients who presented with prolonged ileus were treated conservatively without re-intervention. No mortality occurred during the first postoperative 30 days (Table 4 ).

No difference in age was noted between patients with obstruction and those with peritonitis. However, the numbers of patients with ASA PS classifications III and IV were significantly different $(P=0.015)$. Emergency surgery was defined as an operation within 24 hours after consulting the Department of Surgery.
Table 3. Operative data

\begin{tabular}{lr}
\hline Variable & Value \\
\hline Name of operation & \\
Left hemicolectomy & $2(5.2)$ \\
Anterior resection & $22(57.9)$ \\
Low anterior resection & $5(13.2)$ \\
Segmental resection of colon & $9(23.7)$ \\
Diverting loop ileostomy & \\
Yes & $4(10.5)$ \\
No & $34(89.5)$ \\
Emergency operation & $15(39.5)$ \\
Yes & $23(60.5)$ \\
No & $193.5(100-321)$ \\
Operative time (min)
\end{tabular}

Values are presented as number (\%) or median (range).

Table 4. Short-term outcomes of 38 patients

\begin{tabular}{ll}
\hline Outcome & No. (\%) \\
\hline Postoperative complications & $7(18.4)$ \\
Superficial surgical site infection & $1(2.6)$ \\
Anastomotic leakage & $1(2.6)$ \\
Urine leakage from the ureter anastomotic site & $5(13.2)$ \\
Prolonged ileus & $1(2.6)$ \\
Postoperative bleeding & $1(2.6)$ \\
Pneumonia & $0(0)$ \\
Death in first postoperative 30 days & $15(8-39)$ \\
\hline Hospital stay after surgery (day)
\end{tabular}

Values are presented as number (\%) or median (range).

Seven patients with obstruction and eight patients with peritonitis underwent emergency surgery for their diseases, and this was a statistically significant difference $(\mathrm{P}=0.045)$. Overall postoperative complications were not different between the 2 groups. Superficial surgical site infection occurred more frequently in patients with peritonitis than in those with obstruction $(30.8 \%$ vs. 12.0\%); however, this difference was not statistically significant ( $\mathrm{P}$ $=0.157$ ). One patient with obstruction presented with anastomotic leakage after the first operation, as described earlier. The median operation time was 200 minutes in patients with obstruction and 171 minutes in those with peritonitis. The hospital stays after the first operation were 16 days and 15 days for patients with obstruction and those with peritonitis, respectively, but this difference was not statistically significant $(\mathrm{P}=1.000)$ (Table 5).

\section{DISCUSSION}

Intraoperative colonic irrigation was first introduced by Muir in 
Table 5. Comparison of short-term outcomes in patients with obstruction to those in patients with peritonitis

\begin{tabular}{lccc}
\hline Variable & $\begin{array}{c}\text { Obstruction } \\
(\mathrm{n}=25)\end{array}$ & $\begin{array}{c}\text { Peritonitis } \\
(\mathrm{n}=13)\end{array}$ & P-value \\
\hline Age (yr) & $71(47-92)$ & $79(46-93)$ & 0.196 \\
ASA PS classification, III- IV & 7 & 9 & 0.015 \\
Emergency operation & 7 & 8 & 0.045 \\
Postoperative complications & 8 & 6 & 0.391 \\
Superficial surgical site infection & 3 & 4 & 0.157 \\
Anastomotic leakage & 1 & 0 & 0.465 \\
Prolonged ileus & 4 & 1 & 0.472 \\
Operative time (min) & $200(100-321)$ & $171(146-290)$ & 0.230 \\
Hospital stay (day) & $16(8-23)$ & $15(10-39)$ & 1.000 \\
\hline
\end{tabular}

Values are presented as median (range) or number.

ASA PS, American Society of Anesthesiologists physical status.

1968 [14], and Dudley et al. [15] modified this method and proposed antegrade on-table colonic irrigation with primary anastomosis in 1980. Since the early 1980 s, several studies have supported the feasibility of a one-stage resection and primary anastomosis for patients with unprepared or inadequately prepared bowels who require a resection of a left colon lesion $[5,16,17]$, especially patients with obstruction $[6,18]$ and peritonitis $[19,20]$. The purpose of intraoperative colonic irrigation is to perform a safe anastomosis and decrease the anastomotic leakage rate by reducing the amount of feces around the anastomosis and decompressing the colon $[15,21]$.

Intraoperative colonic irrigation has been used successfully to facilitate primary anastomosis in patients with acute mechanical obstruction of the left colon [21, 22]. In our study, 25 patients $(65.8 \%)$ were included in this category. Of these patients, 21 patients had no obstructive symptom and passed flatus at the time of admission, although the scope could not pass through the malignant lesion in the preoperative colonoscopy finding. However, a distended proximal bowel impacted by watery feces was found during a laparotomy. In these situations, we usually performed intraoperative colonic irrigation with primary anastomosis rather than the Hartmann operation because the obstructive colitis or edema was not severe in the surgical findings. Obstructive colitis sometimes induces mucosal necrosis, which may increase the risk of anastomotic leakage, so an extended resection may be required $[13,18]$. However, a low risk for mucosal necrosis may be expected in this situation because of the relatively short period of obstruction. Many authors reported the usefulness of self-expandable metal stents (SEMSs) in the management of left colonic obstruction as a 'bridge to surgery' $[10,11,23]$. However, insertion of a SEMS can fail about $10 \%$ of the time, and complications, including stent migration, bleeding, and perforation, have been reported [23]. In our institute, we usually discuss with endoscopists the insertion of a SEMS when patients with a complete ma- lignant colon obstruction are admitted. In three patients in our study, insertion of a SEMS failed; one was considered a difficult case, and we were concerned about the risk of bowel perforation when inserting the SEMS. Intraoperative colonic irrigation with primary anastomosis may be an effective method in selected patients for whom insertion of a SEMS failed.

In early studies, the presence of peritonitis was considered a contraindication of primary anastomosis after a colon resection due to high anastomotic leak rates $[24,25]$. However, this concept has been challenged by recent studies [12, 19, 20]. Regenet el al. [19] reported similar mortalities for intraoperative colonic irrigation and primary anastomosis (11\%) compared to the Hartmann operation (12\%) in patients with diverticular perforation. Moreover, the incidence of postoperative complications was higher after the Hartmann operation. Zorcolo et al. [20] reported an anastomotic leakage rate of $5.1 \%$ and a wound infection rate of $4.5 \%$ following primary anastomosis for emergency left colonic and rectal surgery with no difference between patients with obstruction or peritonitis. The presence of peritonitis seems no longer to be an absolute contraindication to primary anastomosis. In contrast, the presence of adverse systemic factors, hemodynamic instability, compromised immunity, and malnutrition can cause anastomotic disruption $[1,12,20]$.

Over the years, many studies have reported on antegrade intraoperative colonic irrigation via the appendix, terminal ileum, or cecum to decompress the fecal loading. These conventional methods of colonic lavage are cumbersome and time consuming and may increase the risk of spillage in the operative field [13]. A new method using a colonic irrigation device with a double lumen was proposed by Park et al. [13] to compensate for these drawbacks. They reported that the wider irrigation catheter would save irrigation time and that the fastening nut and tie of the device would prevent the risk of fecal spillage. The authors also suggested ontable colonoscopy after intraoperative colonic irrigation to detect synchronous polyps. In this study, synchronous polyps were detected in $47 \%$ of the patients, and an extended resection was performed in $17 \%$ of the patients who underwent on-table colonoscopy. Sasaki et al. [18] also detected synchronous polyps in $30.7 \%$ of the patients during on-table colonoscopy after intraoperative colonic irrigation. Moreover, they emphasized that on-table colonoscopy prevented the risk of anastomotic leakage in 2 patients with a necrotic change of the proximal colonic mucosa. However, Otsuka et al. [6] raised a question about the necessity of on-table total colonoscopy because recent advances in radiologic technology and postoperative total colonoscopy may be able to detect synchronous polyps. In our study, on-table colonoscopy was not performed, although we did use a double-lumen device (NICI) for intraoperative colonic irrigation. This was because either the operator did not deem it necessary or colonoscopy was not available due to the odd hour of the surgery. Also, the proximal colon was carefully checked before applying an anvil for anastomosis to exclude the presence of mucosal necrosis in the proximal colon 
and to ensure the safety of the anastomosis.

The rate of wound infection of the primary anastomosis with intraoperative colonic irrigation is generally reported at $4 \%-37 \%$ $[6,13]$. In our study, the wound infection rate was $18.4 \%$ without any significant difference between patients with obstruction and those with peritonitis. In general, intraoperative colonic irrigation seems not to prevent wound infection, which may prolong the postoperative hospital stay. However, all wound infections were classified as grade I according to the Clavien-Dindo classification, and all patients were treated conservatively.

We performed primary anastomosis with intraoperative colonic irrigation in diverse situations that required a left colon resection without an appropriate preoperative mechanical bowel preparation. Although verifying the indications for this procedure was difficult because of the limitations of this retrospective study, we selected another procedure, such as the Hartmann operation, for use with patients with high risk factors, such as severe obstructive colitis, hemodynamic instability, compromised immunity, etc., for anastomotic leakage. Also, a diverting loop ileostomy was conducted in 4 patients who underwent anastomosis below the peritoneal reflection. As suggested by Biondo et al. [12], a protective temporary stoma would be an option in patients with extraperitoneal anastomosis or after technical difficulties.

In conclusion, intraoperative colonic irrigation and primary anastomosis seem safe and feasible in selected patients. Proper selection based on the patient's general condition and the operative findings should reduce the burden of a colostomy in patients who require a left colon resection and who have undergone an inappropriate preoperative mechanical bowel preparation.

\section{CONFLICT OF INTEREST}

No potential conflict of interest relevant to this article was reported.

\section{REFERENCES}

1. Patriti A, Contine A, Carbone E, Gullà N, Donini A. One-stage resection without colonic lavage in emergency surgery of the left colon. Colorectal Dis 2005;7:332-8.

2. Bucher P, Gervaz P, Egger JF, Soravia C, Morel P. Morphologic alterations associated with mechanical bowel preparation before elective colorectal surgery: a randomized trial. Dis Colon Rectum 2006;49:109-12.

3. Zmora O, Wexner SD, Hajjar L, Park T, Efron JE, Nogueras JJ, et al. Trends in preparation for colorectal surgery: survey of the members of the American Society of Colon and Rectal Surgeons. Am Surg 2003;69:150-4.

4. Kehlet H, Büchler MW, Beart RW Jr, Billingham RP, Williamson R. Care after colonic operation--is it evidence-based? Results from a multinational survey in Europe and the United States. J Am Coll Surg 2006;202:45-54.
5. Deans GT, Krukowski ZH, Irwin ST. Malignant obstruction of the left colon. Br J Surg 1994;81:1270-6.

6. Otsuka S, Kaneoka Y, Maeda A, Takayama Y, Fukami Y, Isogai M. One-stage colectomy with intraoperative colonic irrigation for acute left-sided. World J Surg 2015;39:2336-42.

7. Karran SJ. Restoration of intestinal continuity following Hartmann's procedure: the Lothian experience 1987-1992. Br J Surg 1995;82:1138-9.

8. Belmonte C, Klas JV, Perez JJ, Wong WD, Rothenberger DA, Goldberg SM, et al. The Hartmann procedure. First choice or last resort in diverticular disease? Arch Surg 1996;131:612-5.

9. Sabbagh C, Browet F, Diouf M, Cosse C, Brehant O, Bartoli E, et al. Is stenting as "a bridge to surgery" an oncologically safe strategy for the management of acute, left-sided, malignant, colonic obstruction? A comparative study with a propensity score analysis. Ann Surg 2013;258:107-15.

10. Khot UP, Lang AW, Murali K, Parker MC. Systematic review of the efficacy and safety of colorectal stents. Br J Surg 2002;89: 1096-102.

11. van den Berg MW, Sloothaak DA, Dijkgraaf MG, van der Zaag ES, Bemelman WA, Tanis PJ, et al. Bridge-to-surgery stent placement versus emergency surgery for acute malignant colonic obstruction. Br J Surg 2014;101:867-73.

12. Biondo S, Parés D, Kreisler E, Ragué JM, Fraccalvieri D, Ruiz AG, et al. Anastomotic dehiscence after resection and primary anastomosis in left-sided colonic emergencies. Dis Colon Rectum 2005; 48:2272-80.

13. Park UC, Chung SS, Kim KR, Seong MK, Yoon WH, Kim YJ, et al. Single-stage procedure with intraoperative colonoscopy and colonic irrigation in patients with obstructing left-sided colonic cancer. Int J Colorectal Dis 2004;19:487-92.

14. Muir EG. Safety in colinic resection. Proc R Soc Med 1968;61:4018.

15. Dudley HA, Racliffe AG, McGeehan D. Intraoperative irrigation of the colon to permit primary anastomosis. Br J Surg 1980;67:80-1.

16. Pollock AV, Playforth MJ, Evans M. Peroperative lavage of the obstructed left colon to allow safe primary anastomosis. Dis Colon Rectum 1987;30:171-3.

17. Carty NJ, Corder AP, Johnson CD. Colostomy is no longer appropriate in the management of uncomplicated large bowel obstruction: true of false? Ann R Coll Surg Engl 1993;75:46-51.

18. Sasaki K, Kazama S, Sunami E, Tsuno NH, Nozawa H, Nagawa H, et al. One-stage segmental colectomy and primary anastomosis after intraoperative colonic irrigation and total colonoscopy for patients with obstruction due to left-sided colorectal cancer. Dis Colon Rectum 2012;55:72-8.

19. Regenet N, Pessaux P, Hennekinne S, Lermite E, Tuech JJ, Brehant $\mathrm{O}$, et al. Primary anastomosis after intraoperative colonic lavage vs. Hartmann's procedure in generalized peritonitis complicating diverticular disease of the colon. Int J Colorectal Dis 2003;18:503-7.

20. Zorcolo L, Covotta L, Carlomagno N, Bartolo DC. Safety of primary anastomosis in emergency colo-rectal surgery. Colorectal 
Dis 2003;5:262-9.

21. Forloni B, Reduzzi R, Paludetti A, Colpani L, Cavallari G, Frosali D. Intraoperative colonic lavage in emergency surgical treatment of left-sided colonic obstruction. Dis Colon Rectum 1998;41:23-7.

22. Biondo S, Jaurrieta E, Jorba R, Moreno P, Farran L, Borobia F, et al. Intraoperative colonic lavage and primary anastomosis in peritonitis and obstruction. Br J Surg 1997;84:222-5.

23. Sebastian S, Johnston S, Geoghegan T, Torreggiani W, Buckley M.
Pooled analysis of the efficacy and safety of self-expanding metal stenting in malignant colorectal obstruction. Am J Gastroenterol 2004;99:2051-7.

24. Schrock TR, Deveney CW, Dunphy JE. Factor contributing to leakage of colonic anastomoses. Ann Surg 1973;177:513-8.

25. Chappuis CW, Cohn I Jr. Acute colonic diverticulitis. Surg Clin North Am 1988;68:301-13. 